Methods A comprehensive systematic review of HIV and risk behaviour studies in Lebanon was undertaken. Sources of data included (1) PubMed using a strategy with both free text and $\mathrm{MeSH}$ headings, (2) country-level reports and databases including governmental and non-governmental organisations publications, and (3) international organisations reports and databases.

Results Available data indicate that HIV prevalence among the high risk groups ranged between 0 and 3.6\% among men who have sex with men (MSM), 0 and $3.9 \%$ among injecting drug users (IDUs), and $0 \%-0.3 \%$ among female sex workers (FSWs). HIV prevalence in the general population was very limited $(\sim 0 \%)$. Analysis of HIV notified cases indicated a substantial recent increase in the contribution of male same-sex sex, whereby $52 \%$ of notified cases in 2008 were due to MSM transmission compared to $13 \%$ in the cumulative period until 2008. Substantial levels of risk behaviour were reported by the three high-risk groups. MSM reported an average of 9.5 sexual partners in the last year and up to $36 \%$ engaged in male sex work. Seventeen to $65 \%$ of IDUs shared needles/syringes, $33 \%-47 \%$ had sex with FSWs, $17 \%-33 \%$ engaged in sex work, and $24.7 \%$ reported male same-sex sex. FSWs reported an average of 11.7 sexual partners per week. Despite relatively high levels of risk behaviour, condom use in Lebanon was high and among the highest in the MENA region, so were the levels of knowledge of HIV/AIDS and of its modes of transmission. Molecular epidemiology evidence indicated a diverse HIV subtype distribution suggesting multiple exogenous introductions of the virus.

Conclusions The evidence gathered in this review indicates that HIV endemic transmission appears to be still limited in Lebanon with most diagnosed cases representing exogenous exposures. There are however few indications suggesting an emerging epidemic among MSM. HIV surveillance of high-risk groups needs to be actively expanded in Lebanon to track such potential emerging epidemics and prevent further HIV transmission among population groups at high risk of HIV infection.

\section{P1-S1.16 ESTIMATING HIV INCIDENCE RATE AMONG STABLE SEXUAL PARTNERSHIPS IN SUB-SAHARAN AFRICA}

doi:10.1136/sextrans-2011-050108.16

H Chemaitelly, L J Abu-Raddad. Weill Cornell Medical College, Doha, Oatar

Background Empirical evidence suggests that HIV incidence rate within stable discordant sexual partnerships in sub-Saharan Africa (SSA) varies between 1.2 and 19.0 per 100 person-years. Estimating HIV incidence rate within stable discordant partnerships is critical for determining the contribution of HIV sero-conversions among these partnerships to total HIV incidence.

Methods We constructed a mathematical model based on competing-hazards formalism to estimate HIV incidence rate within stable discordant partnerships across 20 countries in SSA. We also used the model to analyse the patterns of HIV discordancy in SSA. The model was parameterised using Demographic Health Survey data and analyses were conducted at endemic equilibrium. Sensitivity analyses were performed to explore the dependence on the dynamical drivers of discordancy.

Results Our model fitted well the empirical epidemiological measures of HIV discordancy and yielded an estimate for HIV incidence rate among discordant partnerships of 11.2 per 100 person-years (95\% CI 8.9 to 13.4 per 100 person-years). HIV incidence rate ranged between 5.5 and 17.2 per 100 person-years across the countries. We also identified HIV incidence rate within stable discordant partnerships and HIV incidence rate from sources external to the partnership (or equivalently HIV prevalence) as key determinants of the variability in discordancy measures across SSA.
Conclusions Our estimate for the HIV incidence rate among discordant partnerships agrees well and falls in the mid-range of empirical estimates for this measure. There is however considerable variability across the countries. Biological and behavioural factors including differences in transmission rates such as due to male circumcision, may have contributed to the variability in HIV incidence rates among discordant partnerships across SSA. More research is needed to elucidate the determinants of this variability in incidence rates.

\section{P1-S1.17 HIV-1 MOLECULAR EPIDEMIOLOGY IN THE MIDDLE EAST AND NORTH AFRICA}

doi:10.1136/sextrans-2011-050108.17

${ }^{1} \mathrm{G}$ Mumtaz, ${ }^{2} \mathrm{~N}$ Hilmi, ${ }^{2} \mathrm{~F}$ A Akala, ${ }^{3} \mathrm{I}$ Semini, ${ }^{4} \mathrm{G}$ Riedner, ${ }^{2} \mathrm{D}$ Wilson, ${ }^{1} \mathrm{~L}$ Abu-Raddad. ${ }^{1}$ Weill Cornell Medical College, Doha, Oatar; ${ }^{2}$ World Bank, USA; ${ }^{3}$ Joint United Nations Programme on HIVIAIDS Regional Support Team, Middle East and North Africa, Egypt; ${ }^{4}$ Regional Office of the Eastern Mediterranean, WHO, Egypt

Background Human Immunodeficiency Virus Type I (HIV-1) is characterised by a high genetic variability. The distribution of HIV1 subtypes in a population can help track transmission patterns and the evolution of the epidemic. The Middle East and North Africa (MENA) continues to be perceived as a region with limited HIV epidemiological data, but recent research indicates that nascent HIV epidemics appear to be emerging among high-risk groups including injecting drug users (IDUs), men who have sex with men (MSM), and female sex workers. The objective of this work was to review all evidence on HIV-1 subtype distribution in MENA where there remains several gaps in our understanding of the HIV epidemic.

Methods A comprehensive systematic review of all HIV-1 molecular epidemiology data in MENA was undertaken. Sources of data included (1) PubMed using a strategy with both free text and $\mathrm{MeSH}$ headings, (2) country-level reports and database including governmental and non-governmental organisations publications, and (3) international organisations reports and databases.

Results In several countries such as in Lebanon, Saudi Arabia, and Yemen, a very diverse distribution of HIV-1 subtypes was observed reflecting principally travel-related exogenous exposures. A trend of a dominant HIV-1 subtype was observed in few other settings and was often linked to HIV transmission within specific high-risk core groups such as subtype A and CRF35_AD among IDUs in Afghanistan, Iran, and Pakistan; and subtype C in heterosexual commercial sex networks in Djibouti and Somalia. Subtype B was predominant in Northern Algeria, Tunisia, and Morocco, but this appeared to reflect a mix of indigenous endemic transmission and exogenous exposures of West European and North American origin.

Conclusions Multiple introductions of HIV-1 variants due to exogenous exposures of nationals seemed common to all MENA countries, as observed from the high diversity in subtypes or the high genetic divergence among any specific subtype even if predominant. This is in part a reflection of the high population mobility in MENA. In several countries though, epidemic-type clustering of specific subtypes suggests established or nascent HIV epidemics among classical core risk groups for HIV infection. With overall weak surveillance systems in MENA, molecular investigations could help identify the emergence of hidden epidemics among high-risk groups. HIV prevention efforts in MENA must be prioritised for these groups. 